\title{
Preparedness for Caesarean section in COVID-19 positive pregnant patient.
}

\author{
Jasiya $\mathrm{MH}^{1}$, ASHOK DEVOOR ${ }^{1}$, and Huded Sridevi ${ }^{1}$ \\ ${ }^{1}$ Bowring and Lady Curzon Hospital
}

July 16, 2020

\begin{abstract}
Introduction : COVID-19 in pregnancy,the outcome is not very clearly defined, this study guides for step wise approach for preparedness if an C-section is planned for COVID-19 positive pregnant patient Aims and objective: * Step wise guide to prepare for C- section for COVID-19 positive pregnant patient Methods : This step wise guide has been formulated after referring guidelines from RCOG,ACOG,FOGSI, and also the SOP followed in our bowring and lady Curzon hospital. Conclusion: Step wise approach allows the surgeon and other health care workers to be adequately prepared both mentally and physically and better and efficient patient care
\end{abstract}

Title page

Title: Preparedness for Caesarean section in COVID-19 positive pregnant patient-A review article

Author: Dr JASIYA AFREEN MH

Co- Author:

1. Dr ASHOOK DEVOOR, Associate professor, department of obstetrics and gynaecology, Bowring and lady Curzon hospital,tasker town, Shivajinagar, Bangalore-01, Karnataka,india

2. Dr HUDED SRIDEVI, head of the department, obstetrics and gynaecology,bowring and lady Curzon hospital,tasker town, Shivajinagar,Bangalore -01,Karnataka,india

Corresponding Author: Dr JASIYA AFREEN MH, Senior resident, department of obstetrics and gynaecology,Address: no 126, F block, Neeladri mahal, Nandidurga road,Jaymahal extension Bangalore-46

Phone number: +91-9591078411

Email address: drjasi14@gmail.com

Running title:

C-section in COVID-19 positive pregnant women

\section{Abstract}

Introduction :

SARS COV-2, also known as COVID-19, first evolved in wuhan city, in china, affecting millions worldwide, On Jan. 30, 2020, the World Health Organization declared the outbreak as a public health emergency of international concern., COVID-19 in pregnancy,the outcome is not very clearly defined, this study guides for step wise approach for preparedness if an C-section is planned for COVID-19 positive pregnant patientAims and objective:* Step wise guide to prepare for C- section for COVID-19 positive pregnant patientMethods : This step wise guide has been formulated after referring guidelines from RCOG,ACOG,FOGSI, and also the 
SOP followed in our bowring and lady Curzon hospital.Conclusion:Step wise approach allows the surgeon and other health care workers to be adequately prepared both mentally and physically, and would enable to improved and efficient patient care .

\section{Introduction:}

SARS COV-2, also known as COVID-19, first evolved in wuhan city, in china,affecting millions worldwide, On Jan. 30, 2020, the World Health Organization declared the outbreak as a public health emergency of international concern.

\section{Importance of COVID-19 in pregnancy:}

- During pregnancy, women experience immunologic and physiologic changes that could increase their risk for more severe illness from respiratory infections $(1,2)$

- Symptomatic pregnant and nonpregnant women with COVID-19 reported similar frequencies of cough $(>50 \%)$ and shortness of breath (30\%), but pregnant women less frequently reported headache, muscle aches, fever, chills, and diarrhea.(mmwstudy)

- Preliminary experience with pregnant patients with COVID-19 suggests that the clinical outcome of the mother and neonate is often favorable, but it is unclear how and when these patients gave birth. ${ }^{3}$

- The presence of COVID-19 in a pregnant patient raises concerns, as other types of coronaviruses were frequently associated with adverse outcomes ${ }^{4}$

- Covid 19 in pregnancy has not been known to cause severe disease,though has increased risk of contracting disease due to immunocompromised state in pregnancy

- Currently, it isn't clear if pregnant women have a greater chance of getting sick from the COVID19 virus or if they are more likely to experience serious illness ${ }^{5}$

- Because coronavirus disease 2019 might increase the risk for pregnancy complications, management should optimally be in a health care facility with close maternal and fetal monitoring. ${ }^{6}$

- Authorities and professional societies, such as the Italian Health Council, ${ }^{7}$ the English Royal College of Obstetricians and Gynaecologists, ${ }^{8}$ have taken a stance that COVID-19 is not a contraindication to vaginal delivery.

\section{Indications of C- section in COVID-19 pregancy}

1)obstetric indication only

2) If pregnancy has to be terminated in case of acute SARS,

\section{Methods:}

Well preparedness forms the basis of any successful procedure

\section{Step-1 :}

- OT area should be well defined

- There should be separate areas each for Donning and Doffing

- There should be access to take shower post OT,after Doffing.

- There should be separate entry for staff and for patient into the OT complex

- Next, we can proceed towards step -2

\section{Step-2:}

A team should be formed comprising of minimum number of staff to avoid exposure,

1. Operating surgeon

2. Assisting surgeon-1

3. Anaesthesist

4. Assisting staff nurse

5. Group D/ helper 
6. Paediatrician \#None of the other staff are allowed to enter OT complex. \#One doctor should be on call, if any help or extra hands required.

\section{Step-3:}

Indication for C- section:

1)Strictly for obstetric indications

2)If required, for SARI cases

\section{Step-4}

OT-

- Adequate light for visualization

- To check for appropriate functioning of equipments required for proedures

- Drugs to be checked, with expiry date,required for anaesthesia/ obstetrics

- No AC

- Disposable drapes- if possible

- Minimum instruments to avoid unnecessary exposure

- Anaesthesia as per anaesthesist convenience

- Donning with PPE, and then OT gown to be worn.

\section{Step-5}

Pre op prerequisites:

1. Consent- A consent should be taken at the time of admission,to avoid delay if emergency is planned

2. Preparations- parts preparation

3. Medications- antibiotic after test dose and antacid

\section{Donning:}

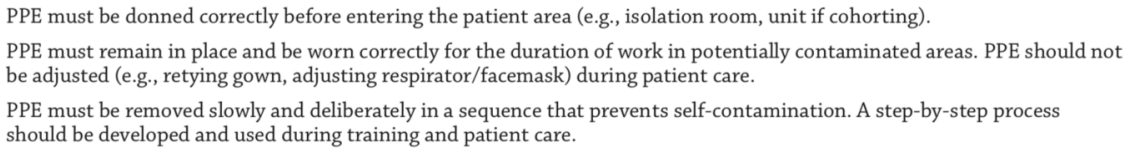

\section{Step wise donning}

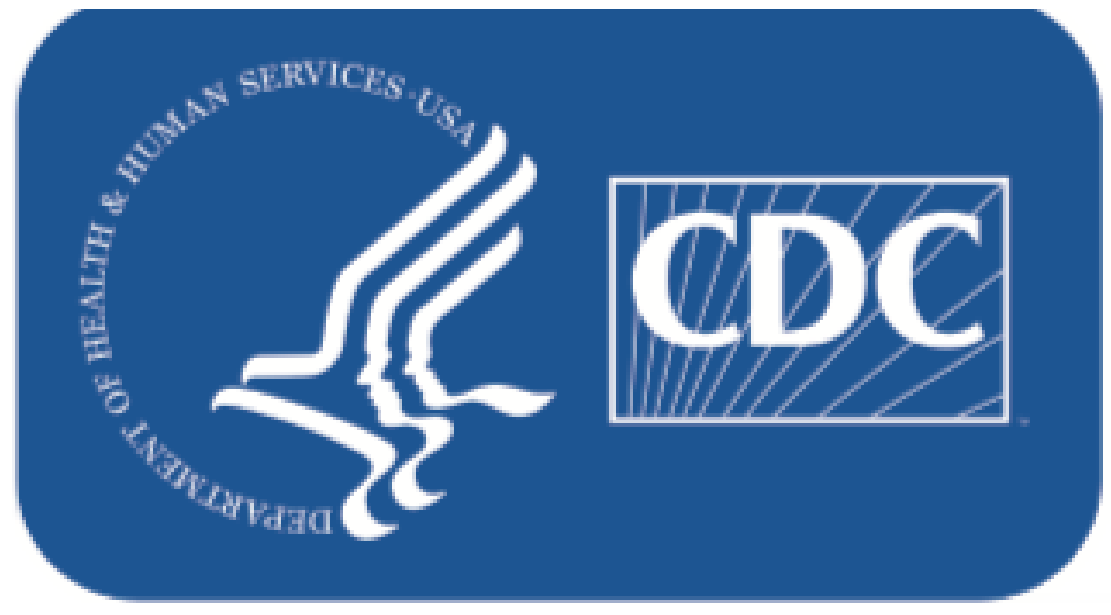




\section{Donning (putting on the gear):}

More than one donning method may be acceptable. Training and practice using your healthcare facility's procedure is critical. Below is one example of donning.

1. Identify and gather the proper PPE to don. Ensure choice of gown size is correct (based on training).

2. Perform hand hygiene using hand sanitizer.

3. Put on isolation gown. Tie all of the ties on the gown. Assistance may be needed by another HCP.

4. Put on NIOSH-approved N95 filtering facepiece respirator or higher (use a facemask if a respirator is not available).

If the respirator has a nosepiece, it should be fitted to the nose with both hands, not bent or tented. Do not pinch the nosepiece with one hand. Respirator/facemask should be extended under chin. Both your mouth and nose should be protected. Do not wear respirator/facemask under your chin or store in scrubs pocket between patients.*

» Respirator: Respirator straps should be placed on crown of head (top strap) and base of neck (bottom strap). Perform a user seal check each time you put on the respirator.

"Facemask: Mask ties should be secured on crown of head (top tie) and base of neck (bottom tie). If mask has loops, hook them appropriately around your ears.

5. Put on face shield or goggles. When wearing an N95 respirator or half facepiece elastomeric respirator, select the proper eye protection to ensure that the respirator does not interfere with the correct positioning of the eye protection, and the eye protection does not affect the fit or seal of the respirator. Face shields provide full face coverage. Goggles also provide excellent protection for eyes, but fogging is common.

6. Put on gloves. Gloves should cover the cuff (wrist) of gown.

7. HCP may now enter patient room.

\section{After donning, sanitise hands and surgical scrub to be worn, and surgical gloves.}

Procedure;

- After anaesthesia, patient in supine position

- Under all aseptic precautions, foleys catherization done

- Parts painted and draped

- Pfannelsteil incision given, abdomen opened in layers,

- Peritoneum opened,

- Kerrs incision given on uterus, allow the suction tube to be at the incision site, to avoid spilling of amniotic fluid, baby is then extracted

- Cord clamping and cutting done and handed over to paediatrician

- Allow placenta to separate on its own, to avoid unnecessary splashing the blood

- Uterus closed in 1 or 2 layers

- Peritoneum closed

- Rectus sheath closed

- Skin closed with s/c,matress/staples

Post-Op medications:

- Fluids as required 
- Antacid

- Antibiotic

- Analgesic - NSAIDs to be avoided

- Check for bleeding

- Foleys Catheter could be discarded after $24 \mathrm{hrs}$ if favourable

- Advised for early ambulation

\section{Doffing}

Remove the apron, surgical scrub, and gloves while leaving OT

Disinfect, and then Doffing

\section{Doffing (taking off the gear):}

More than one doffing method may be acceptable. Training and practice using your healthcare facility's procedure is critical. Below is one example of doffing.

1. Remove gloves. Ensure glove removal does not cause additional contamination of hands. Gloves can be removed using more than one technique (e.g., glove-in-glove or bird beak).

2. Remove gown. Untie all ties (or unsnap all buttons). Some gown ties can be broken rather than untied. Do so in gentle manner, avoiding a forceful movement. Reach up to the shoulders and carefully pull gown down and away from the body. Rolling the gown down is an acceptable approach. Dispose in trash receptacle.*

3. HCP may now exit patient room.

4. Perform hand hygiene.

5. Remove face shield or goggles. Carefully remove face shield or goggles by grabbing the strap and pulling upwards and away from head. Do not touch the front of face shield or goggles.

6. Remove and discard respirator (or facemask if used instead of respirator).* Do not touch the front of the respirator or facemask.

»Respirator: Remove the bottom strap by touching only the strap and bring it carefully over the head. Grasp the top strap and bring it carefully over the head, and then pull the respirator away from the face without touching the front of the respirator.

» Facemask: Carefully untie (or unhook from the ears) and pull away from face without touching the front.

7. Perform hand hygiene after removing the respirator/facemask and before putting it on again if your workplace is practicing reuse.

\section{Step-6}

OT -

- Proper handling of the waste

- All the instruments to be autoclaved 
- OT to be fumigated

Post-op concerns:

- Patient should be advised for early ambulation

- SPO2 and TPR charting daily

- Resumption of step wise diet after $24 \mathrm{hrs}$

- Plenty of oral fluids and adequate hydration

- Vitamin c and zinc supplements

- Breast feeding is not contraindicated, but as such a new born is kept in NICU for monitoring, as there is risk of transmitting infection while handling during breastfeeding

- Suture removal as per protocol

- Swab for the newborn advised

- If asymptomatic, then swab could be repeated on 10 th day/ or discharged with advise of home isolation for another 14 days.

\section{Conclusion :}

COVID-19 in pregnancy can be managed with early diagnosis and treatment,C- section is indicated for obstetrics reasons only and if SARI cases requiring immediate ventilator, step wise approach allows the surgeon and as well as other health care workers to be adequately prepared both mentally and physically and better and efficient patient care can be provided.

- Disclosure of interest: No conflicts to be disclosed, includes financial, personal,political, intellectual,or religious.

- No funding has been sponsored by any organization

- Contribution to authorship:

1. Dr Jasiya mh- has been working on this topic since 1 month, studying various guidelines and forming a cumulative protocol to help and benefit other surgeons to carry out hastle free $\mathrm{C}$ section, after being posted to covid duty, implementing the same protocol and assessing to see the outcome, which was beneficial.

2. Dr Ashok Devoor- has been helping in various ways to guide,monitoring and scrutnising and helping to get access to information, he has been assisting in every step to get a succesfull outcome.

3. Dr Huded Sridevi- has been a strong support, she has been improvising the article and giving the necessary corrections, she has been very encouraging and motivating.

Bibiliography

1)1,2-Della Gatta A, Rizzo R, Pilu G, Simonazzi G. Coronavirus disease 2019 during pregnancy: a systematic review of reported cases. American journal of obstetrics and gynecology. 2020 Jul;223(1):36-41.

3)Chen L, Li Q, Zheng D, Jiang H, Wei Y, Zou L, Feng L, Xiong G, Sun G, Wang H, Zhao Y. Clinical characteristics of pregnant women with Covid-19 in Wuhan, China. New England Journal of Medicine. 2020 Apr 17.

4)Ramsey PS, Ramin KD. Pneumonia in pregnancy. Obstet Gynecol Clin North Am 2001;28:553-69.

5)Rasmussen SA, Kissin DM, Yeung LF, et al.; Pandemic Influenza and Pregnancy Working Group. Preparing for influenza after 2009 H1N1: special considerations for pregnant women and newborns. Am J Obstet Gynecol 2011;204(Suppl 1):S13-20

6) World Health Organization Coronavirus disease (COVID-19) outbreak. https://www.who.int/emergencies/diseases/novel-coronavirus-2019 Available at:

7). Royal College of Obstetricians \& Gynaecologists Coronavirus (COVID-19) infection in pregnancy. Information for healthcare professionals. $\quad$ Version 8. 
2020. https://www.rcog.org.uk/globalassets/documents/guidelines/2020-04-17-coronavirus-covid-19infection-in-pregnancy.pdf Available at:

8)Di Mascio D., Khalil A., Saccone G. Outcome of coronavirus spectrum infections (SARS, MERS, COVID19) during pregnancy: a systematic review and meta-analysis. Am J Obstet Gynecol MFM. 2020 